\title{
Minimum 10-year follow-up of endovascular repair for acute traumatic transection of the thoracic aorta
}

\author{
Ludovic Canaud, MD, PhD, Charles Marty-Ané, MD, PhD, Vincent Ziza, MD, \\ Pascal Branchereau, MD, and Pierre Alric, MD, PhD
}

Objective: Thoracic endovascular aortic repair (TEVAR) for traumatic rupture of the descending thoracic aorta seems, in the short term, to be associated with better outcomes than open repair, but long-term data are lacking.

\begin{abstract}
Methods: A review was conducted of a prospectively maintained database of patients who underwent TEVAR for traumatic rupture of the descending thoracic aorta in our unit, with a minimum 10-year follow-up. Follow-up computed tomography scans were performed at 1 week, 3 and 6 months, and annually thereafter. Particular attention was focused on device-related issues.
\end{abstract}

Results: Among the 53 patients, 17 had a minimum 10-year follow-up: mean age was $45.8 \pm 17$ years (range: 18-78 years); 4 were women. Mean follow-up was 11.6 years (range: 10.1-13.1 years). Technical success was achieved in $100 \%$ of cases. The distribution of the proximal landing zone was zone 2 in 4 cases, zone 3 in 13 cases. A case of inadvertent coverage of supra-aortic trunks occurred intraoperatively. An early proximal type I endoleak was successfully treated by proximal implantation of an additional second stent-graft. No perioperative death was observed, and none of the patients suffered transient or permanent paraplegia, or cerebral complication. After a minimum 10-year follow-up, all patients were still alive. Follow-up computed tomography scans did not reveal any stent-graft migration or collapse, or secondary endoleaks. However, we observed that the proximal and distal aortic neck dilated to some extent, as is the natural history of the thoracic aorta. This dilation was more marked in patients aged $<30$ years.

Conclusions: Our minimum 10-year follow-up study of endovascular repair for acute traumatic transection of the thoracic aorta demonstrated that the reduction in the operative mortality rate of TEVAR, compared with open repair, lasts over time, without any device-related issues. Longer-term follow-up is necessary to determine whether the thoracic aorta expansion continues and becomes clinically significant. (J Thorac Cardiovasc Surg 2015;149:825-9)

See related commentary on pages 829-30.

The treatment of traumatic transection of thoracic aorta has seen many changes over the past 20 years. Thoracic endovascular aortic repair (TEVAR) has been rapidly adopted as an alternative to the traditional open repair for treatment of these lesions. Two prospective observational studies from the American Association for the Surgery of Trauma (AAST), evaluating the management and outcomes of blunt thoracic artery injury studies, demonstrated a marked change in operative management, from exclusively open repairs $(100 \%)$ in the early part of that period, to a majority of endovascular repairs $(65 \%)$.

\footnotetext{
From the Department of Thoracic and Vascular Surgery, Arnaud de Villeneuve Hospital, Montpellier, France.

Disclosures: Authors have nothing to disclose with regard to commercial support. Received for publication Aug 22, 2014; revisions received Oct 19, 2014; accepted for publication Oct 26, 2014; available ahead of print Dec 3, 2014.

Address for reprints: Ludovic Canaud, MD, $\mathrm{PhD}$, Service de Chirurgie Vasculaire et Thoracique, Hôpital A deVilleneuve, 191 av Doyen Gaston Giraud, 34090 Montpellier, France (E-mail: ludoviccanaud@hotmail.com).

$0022-5223 / \$ 36.00$

Copyright $(2015$ by The American Association for Thoracic Surgery

http://dx.doi.org/10.1016/j.jtcvs.2014.10.113
}

This paradigm shift has improved the mortality rate in these patients, as demonstrated by the second AAST study $^{2}: 7.2 \%$ for endovascular repair versus $23.5 \%$ for open repair. Stent grafting has become the first-line approach for traumatic thoracic aortic transections. Although many early reports are overwhelmingly positive, concerns have been raised regarding the long-term durability of this approach in these young patients. Long-term follow-up data are clearly critical to assess the durability of TEVAR in this younger population of patients, who have longer life expectancies than patients with aneurysmal disease. Material failures, such as stent fractures and fabric fatigue, may become more significant during ensuing decades of follow-up. Because the aorta tends to dilate with age, smaller-sized devices appropriate at the time of implantation may lose their fixation over time. Therefore, evaluation of long-term device performance in this disease-specific condition is of high importance. The purpose of this study was to evaluate outcomes of endovascular repair of traumatic thoracic aortic rupture in our unit, with a minimum 10-year follow-up.

\section{METHODS}

Ethical approval was obtained from the scientific and research ethics board of our hospital. 


\section{Abbreviations and Acronyms \\ AAST $=$ American Association for the Surgery of Trauma \\ $\mathrm{CT}=$ computed tomography \\ LCCA $=$ left common carotid artery \\ LSA $=$ left subclavian artery \\ OR $=$ odds ratio \\ TEVAR $=$ thoracic endovascular aortic repair}

\section{Patients}

A review of a prospectively maintained database of patients who underwent TEVAR for traumatic rupture of the descending thoracic aorta in our unit, with a minimum 10-year follow-up, was performed. Early and longterm outcomes were assessed. Diagnosis of aortic disruption was achieved by a preprocedural contrast-enhanced computed tomography (CT) scan of all patients.

\section{Endovascular Repair}

Suitable morphology for stent-graft placement requires a proximal aortic neck length of at least $20 \mathrm{~mm}$ between the ostium of the left common carotid artery (LCCA) and the tear. Measurements from preprocedural imaging data were used to select the appropriate diameter and length of the stentgraft. Devices were sized to be $10 \%$ to $20 \%$ greater than the minor axis of the aortic neck. This oversizing may have been greater in these patients, owing to the unavailability of small $(<26 \mathrm{~mm})$ thoracic devices at that time. All procedures were done with the patient under general anesthesia. Cerebrospinal fluid drainage was not used for any patient.

Patients were prospectively monitored by scheduled clinical observation and with contrast-enhanced CT, preoperatively, before hospital discharge, at $1,3,6$, and 12 months, and annually thereafter. CT images were analyzed on a 3-dimensional workstation (Centricity, General Electric, Emporium, Pa). A comparison was made for each patient at each postoperative scan, and an annualized rate of change ( $\mathrm{mm}$ per year) was calculated by dividing the difference in diameter ( $\mathrm{mm}$ ) by the number of intervening months and multiplying by 12 . All measurements were then expressed as the mean \pm standard deviation. The first postoperative scan at 1 month was considered the baseline, to try to negate the perioperative effect of the stent-graft on the neck. All aortic CT measurements were taken in a perpendicular plane using centerline reconstructions. Migration was defined as $>5 \mathrm{~mm}$ movement of the stent-graft relative to the left subclavian artery (LSA), or any movement requiring a secondary procedure. Proximal and distal aortic neck diameter was measured $10 \mathrm{~mm}$ above and below the stent-graft in planes orthogonal to the aorta. The inner diameter of the aortic neck was measured on the major and minor axis.

\section{Statistical Analysis}

Categoric data are presented as counts (percentage); continuous data are presented as mean \pm standard deviation, or median with interquartile range. Categoric variables were compared using a $\chi^{2}$ analysis for normally distributed data, and the Fisher exact test for nonparametric data. Continuous data were analyzed using the Student $t$ test. Statistical analyses were performed using SAS software (version 9.1; SAS Institute Inc, Cary, NC); $P<.05$ was considered significant.

\section{RESULTS}

\section{Perioperative Data}

At our institution, between January 2001 and January 2014, 53 consecutive patients underwent TEVAR for acute traumatic rupture of the aortic isthmus. Of these, 17 had a minimum 10-year follow-up. Median follow-up was 11.7 years (range: 10-13.2 years). Clinical follow-up data were available for all patients.

Patients included 4 women and 13 men, with a mean age of $45.8 \pm 17$ years (range: $18-78$ years). The mean injury severity score was $36 \pm 6$. The grades of aortic injury were: grade 2 (intramural hematoma; $\mathrm{n}=8$ ); grade III (pseudoaneurysm; $\mathrm{n}=7$ ); and grade IV (free rupture; $\mathrm{n}=2$ ). The mean delay between the time of aortic disruption and endovascular treatment was $5.2 \pm 7.8$ days. No patients died during the interval between diagnosis and TEVAR, and no patients underwent open repair during the period of inclusion. Three endovascular devices were used: the Excluder Tag (W. L. Gore \& Associates, Flagstaff, Ariz; $\mathrm{n}=7$ ); the Talent (Medtronic Vascular, Santa Rosa, Calif; $\mathrm{n}=9$ ); and the Zenith distal extension cuff (Cook Inc, Bloomington, Ind; $n=1$ ). The distribution of the proximal landing zone was zone 2 in 4 cases, zone 3 in 13 patients. The LSA was covered in 4 cases.

In 3 patients, for whom the proximal aortic neck length was insufficient, the ostium of the LSA was deliberately covered. For 1 patient whose hemodynamic status was stable at the time of diagnosis, the landing zone was extended by prophylactic LSA to the LCCA transposition before endovascular repair. For 1 patient in whom the hemodynamic status was unstable at the time of aortic disruption, an LSAto-LCCA transposition was secondarily performed to treat vertebrobasilar insufficiency (vertigo and drop attacks).

Median aortic neck diameter, at the time of stent-graft placement, was $21.5 \mathrm{~mm}$ (range: $16-31 \mathrm{~mm}$ ), and median nominal device diameter was $28.4 \pm 3 \mathrm{~m}$ (range: $22-40$ $\mathrm{mm})$, resulting in a mean oversizing of $25 \% \pm 9 \%$. The mean distal aortic diameter was $19.8 \pm 4.5 \mathrm{~mm}$. The mean stent-graft length was $104 \pm 27 \mathrm{~mm}$ (range: 100-150 mm).

\section{Clinical Outcomes}

Perioperative results. Technical success was achieved in all cases. In 4 patients, for whom proximal aortic neck length was insufficient, the ostium of the LSA was deliberately covered. Intraoperatively, a proximal stent-graft migration occurred, totally covering the LCCA ostium. The stent-graft was pulled distally by traction, using an inflated low-pressure balloon, and re-established flow to the left common carotid artery. However, an excessive distal migration led to a proximal type I endoleak. This endoleak was successfully treated by a proximal implantation of a second stent-graft on the second postoperative day. No perioperative death was observed, and none of the patients suffered transient or permanent paraplegia, or cerebral complication.

Long-term results: Minimum 10-year follow-up. At a minimum 10-year follow-up, all the patients were still alive. None of them required open surgical conversion, or 


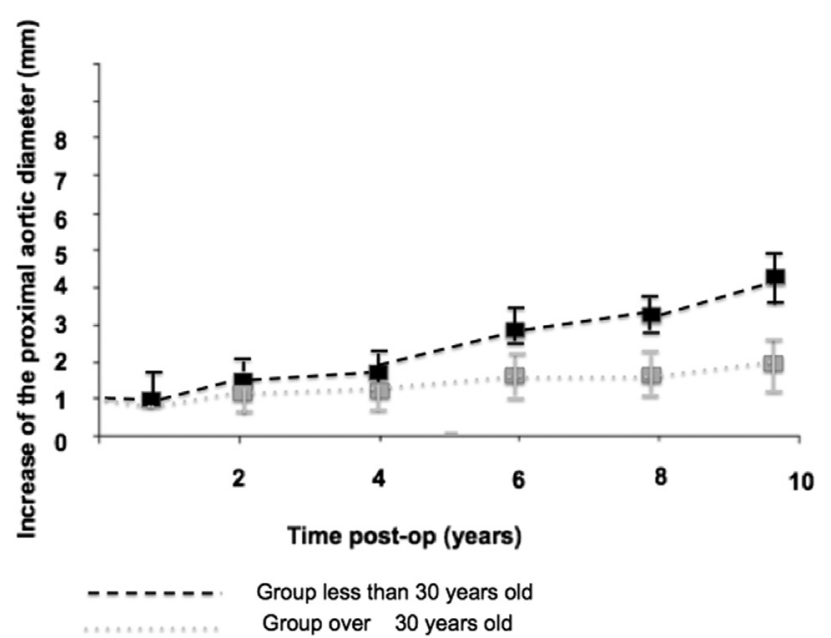

FIGURE 1. Rate of growth of aortic neck (mean \pm SD) in patients aged $<30$ years (group I) versus patients aged $>30$ years (group II).

secondary endovascular procedures during follow-up. Follow-up CT scans did not disclose any stent-graft migration or collapse, or a secondary endoleak. No strokes or paraplegia occurred during follow-up.

\section{Proximal and Distal Thoracic Aorta Evolution}

During the 10 years of follow-up, of the 17 patients included, 11 patients had undergone all the yearly CT follow-up scans. All the patients had at least $6 \mathrm{CT}$ scans during follow-up, including at least $1 \mathrm{CT}$ scan performed 10 years after the TEVAR.

Proximal neck diameter trend. The neck diameter was observed to increase during the postoperative period. At 10 years, the net increase in neck diameter, relative to the first postoperative scan, was $3.3 \pm 1.5 \mathrm{~mm}$. As shown in Figure 1, the aorta dilated consistently throughout the 10year period. Patients were divided into 2 groups according to their age ( $<30$ years vs $>30$ years). We choose 30 years of age as the dividing point because we wanted to have 2 comparable groups in terms of number of patients: 4 patients were $<30$ years old; 7 patients were $>30$ years old. Additionally, during collection of the data, an important difference in neck diameter increase was observed between young and elderly patients. At 10 years, in the younger group of patients, the net increase in neck diameter was significantly greater than that in the older patients: $P=.0037 ; 4 \pm 1.2 \mathrm{~mm}$ versus $1.5 \pm 1.7 \mathrm{~mm}$.

Distal neck diameter trend. The neck diameter was observed to increase during the postoperative period. At 10 years, the net increase in neck diameter, relative to the first postoperative scan, was $2.7 \pm 1 \mathrm{~mm}$. At 10 years, the net increase in neck diameter demonstrated a tendency to be greater in the group of patients $<30$ years old, but this difference failed to reach statistical significance: $P=.065,3.3 \pm 1.6 \mathrm{~mm}$ versus $2.1 \pm 1.6 \mathrm{~mm}$.

\section{Statistical Analysis}

We did not find any impact of device type, patient gender, or proximal landing zone on the rate of aortic growth. However, interpretation of these results is limited by the small size of the sample.

\section{DISCUSSION}

The use of TEVAR to treat patients with acute traumatic transection of the descending aorta has dramatically affected the outcomes of these critically injured patients. Results in the current literature, ${ }^{3-5}$ and the expanding clinical use of thoracic stent-grafts for this indication, consistently display a survival advantage of endovascular repair, as well as a reduction in major perioperative morbidity, including paraplegia. In 2008, the AAST study group provided an update ${ }^{2}$ since the inception of TEVAR. A comparison was conducted of 193 patients (125 TEVAR; 68 open surgery) injured in the period from 2005 to 2007. They found no differences in the systemic complication rate but a significant reduction in gross mortality rate (odds ratio [OR] 3.97; $P=.001$ ) with TEVAR compared with open repair. After adjusting for the degree of injury (Glasgow Coma Score, hypotension, and extrathoracic injury), TEVAR provided clear attenuations in mortality rate (open repair vs TEVAR; OR 8.4; $P<.0001$ ), and in the transfusion requirements (open repair vs TEVAR; OR $4.98 ; P=.046$ ) accompanying open repair.

The debate regarding delay of treatment in those with other significant injuries is dampened by the use of the stent-graft. TEVAR can be performed just before or immediately after other necessary procedures with minimal compounded stress. Still, should delay seem prudent because of other critical needs, TEVAR can be accomplished on a delayed basis as well.

TEVAR is not without its shortcomings. For one, it cannot be emphasized enough just how critical the endovascular operating team and procedure facility are to quality outcomes. Appropriate endovascular inventories, imaging, and aroundthe-clock accessibility are crucial in making this unique trauma therapy available. An important limitation for TEVAR in the trauma population is aortic anatomy. As most of the patients sustaining traumatic aortic injury are young, the size of their aortas is relatively small. A significant proportion have aortas adjacent to the injury that are smaller than $20 \mathrm{~mm}$. Oversizing of thoracic stent-grafts has unmasked serious issues with graft infolding, graft collapse, and graft failure., Thoracic stent-grafts should, therefore, be oversized by only roughly $10 \%$ of the aortic diameter. However, the smallest-diameter thoracic stent-grafts available are $21 \mathrm{~mm}$.

In addition to the small aortic diameter, another aspect of aortic morphology critical to proper stent-graft placement is the arch configuration. The posterior aortic arch genu creates an angulation along the inner aortic curvature such that the proximal edge of the stent-grafts may not conform 
to the aortic wall at this position, which is also called the "bird's beak" deformity. This, in and of itself, can lead to graft collapse, migration, and endoleak, in this extremely high-flow location. We recently assessed the impact of stent-graft design (first vs last generation of thoracic stent-grafts) on the outcome of endovascular repair of acute traumatic thoracic aortic transection. ${ }^{8}$ We have clearly demonstrated that the enhanced stent-graft conformability ${ }^{9}$ and more-accurate delivery systems have significantly decreased the morbidity of endovascular repair of acute traumatic transection of the thoracic aorta.

An area of remaining concern regarding the long-term follow-up of TEVAR is that few studies report late outcomes. Our minimum 10-year follow-up study of endovascular repair for acute traumatic transection of the thoracic aorta demonstrated that the reduction in the operative mortality rate of TEVAR, compared with open repair, lasts over time, without any device-related issues. The expected durability of any endovascular aortic repair depends on the life expectancy of the patient and the long-term behavior of the aortic segment in question. The morphologic changes of the aorta that come with age may still occur in the late phases, and may lead to stent-graft-related complications. It has been well documented that infrarenal aortic dilatation occurs after endovascular aneurysm repair, and can result in graft migration and type I endoleaks. ${ }^{10}$ A recent study reviewed 1000 thoracic CT scans to assess the natural behavior of the thoracic aorta with increasing age. ${ }^{11}$ Between teenagers and octogenarians, a 1-cm increase was observed in the mean diameter at the thoracic aortic isthmus. The radial force imparted to the aorta by the stent-graft could be expected to increase the rate of dilatation of the aorta. Continuing expansion of aortic necks poses a substantial threat to the long-term durability of TEVAR, because proximal fixation is jeopardized once the diameter of the neck exceeds that of the stent-graft. If this dilatation, seen in nonstented aortas, occurred to this degree after TEVAR, there would be a risk of type I endoleaks, device migration, and collapse.

In our study, we observed that the proximal and distal aortic neck dilated to some extent, as is the natural history of the thoracic aorta. Additionally, an increased growth rate in the area of the proximal aortic neck compared with the distal aortic neck was observed. The thoracic aorta significantly dilated at a faster rate in the group of patients aged $<30$ years. To date, thoracic aortic dilatation has not been clinically significant. These findings corroborate the observation that aortic expansion seems to be more related to the natural history of the thoracic aorta than to any effect of the stent-graft.

One important aspect of the follow-up of these young patients is adherence to radiologic follow-up. One reason for the positive follow-up in our study may be that none of the patients came from a catchment area far from our centers. The very encouraging results of this approach, even after long term-follow-up, have prompted us to change our surveillance protocol to decrease the radiation dosage. During the first 3 years after TEVAR, patients have an annual CT scan; after that, they have a low-dose CT scan every 2 years.

This study is not without limitations. Although the data were prospectively collected, it is retrospective, and it is statistically limited because of the small number of patients enrolled. Another shortcoming of this study is that the preoperative aortic diameters before the injury could be overestimated. The first postoperative CT scan, with stent-graft in place, was deliberately chosen as the baseline because preoperative scans reflect any hemodynamic instability in injured patients. Differences in aortic diameters of up to $30 \%$ between preoperative and postoperative CT scans have been documented in such hypotensive trauma patients. ${ }^{12}$

\section{CONCLUSIONS}

Our minimum 10-year follow-up study of endovascular repair for acute traumatic transection of the thoracic aorta demonstrated that the reduction in the operative mortality rate of TEVAR, compared with open repair, lasts over time, without any device-related issues. However, in the present study, we observed that the proximal and distal aortic neck dilated to some extent, as is the natural history of the thoracic aorta. This change was more pronounced in patients aged $<30$ years. Longer-term follow-up is necessary to determine whether this expansion continues and becomes clinically significant.

\section{References}

1. Fabian TC, Richardson JD, Croce MA, Smith JS Jr, Rodman G Jr, Kearney PA, et al. Prospective study of blunt aortic injury: Multicenter Trial of the American Association for the Surgery of Trauma. J Trauma. 1997;42:374-80.

2. Demetriades D, Velmahos GC, Scalea TM, Jurkovich GJ, Karmy-Jones R, Teixeira PG, et al. Operative repair or endovascular stent graft in blunt traumatic thoracic aortic injuries: results of an American Association for the Surgery of Trauma Multicenter Study. J Trauma. 2008;64:561-70.

3. Canaud L, Alric P, Branchereau P, Joyeux F, Hireche K, Berthet JP, et al. Open versus endovascular repair for patients with acute traumatic rupture of the thoracic aorta. J Thorac Cardiovasc Surg. 2011;142:1032-7.

4. Piffaretti G, Benedetto F, Menegolo M, Antonello M, Tarallo A, Grego F, et al. Outcomes of endovascular repair for blunt thoracic aortic injury. J Vasc Surg. 2013;58:1483-9.

5. Khoynezhad A, Azizzadeh A, Donayre CE, Matsumoto A, Velazquez O, White R, et al. Results of a multicenter, prospective trial of thoracic endovascular aortic repair for blunt thoracic aortic injury (RESCUE trial). J Vasc Surg. 2013; 57:899-905.

6. Canaud L, Alric P, Desgranges P, Marzelle J, Marty-Ané C, Becquemin JP. Factors favoring stent-graft collapse after thoracic endovascular aortic repair. J Thorac Cardiovasc Surg. 2010;139:1153-7.

7. Muhs BE, Balm R, White GH, Verhagen HJ. Anatomic factors associated with acute endograft collapse after Gore TAG treatment of thoracic aortic dissection or traumatic rupture. J Vasc Surg. 2007;45:655-61.

8. Canaud L, Joyeux F, Berthet JP, Hireche K, Marty-Ané C, Alric P. Impact of stent-graft development on outcome of endovascular repair of acute traumatic transection of the thoracic aorta. J Endovasc Ther. 2011;18: 485-90.

9. Canaud L, Cathala P, Joyeux F, Branchereau P, Marty-Ané C, Alric P. Improvement in conformability of the latest generation of thoracic stent grafts. $J$ Vasc Surg. 2013;57:1084-9. 
10. Litwinski RA, Donayre CE, Chow SL, Song TK, Kopchok G, Walot I, et al. The role of aortic neck dilation and elongation in the etiology of stent graft migration after endovascular abdominal aortic aneurysm repair with a passive fixation device. J Vasc Surg. 2006;44:1176-81.

11. Hartley MC, Langan EM III, Cull DL, Taylor SM, Carsten CG III, Blackhurst DW. Evaluation of the diameter of the proximal descending thoracic aorta with age: implications for thoracic aortic stent grafting. Ann Vasc Surg. 2009;23:639-44.

12. van Prehn J, van Herwaarden JA, Muhs BE, Arnofsky A, Moll FL, Verhagen HJ. Difficulties with endograft sizing in a patient with traumatic rupture of the thoracic aorta: the possible influence of hypovolemic shock. J Vasc Surg. 2008; 47:1333-6.

\title{
EDITORIAL COMMENTARY
}

\section{Burning bridges: Endovascular therapies for acute traumatic aortic transection}

\author{
Grayson H. Wheatley III, MD
}

See related article on pages 825-9.

Endovascular repair of acute aortic transection has often been viewed as a bridge to a more definitive open repair, particularly in younger patients. The less-invasive nature of endovascular aortic stent procedures has enabled acutely injured patients to undergo repair of their aorta while the remainder of their injuries are triaged. Concern regarding the durability of the aortic stent-grafts, along with potential for aortic dilation or growth in young patients, has prompted many surgeons to suggest a potential need for a definitive open surgical procedure after thoracic endovascular aortic repair (TEVAR) for traumatic rupture of the descending thoracic aorta, once patients have fully recovered from their injuries. ${ }^{1}$

In this issue of the Journal, Canaud and colleagues ${ }^{2}$ temper those concerns by providing 10-year follow-up data in patients who received TEVAR for acute traumatic transection. Convincing data show that TEVAR in the acute setting improves outcomes in traumatic aortic transection (TAT) patients. ${ }^{3}$ The current study is one of the first to evaluate the longer-term outcomes in this patient population. A total of 17 patients ( 4 women and 13 men, with an age range

\footnotetext{
From the Division of Cardiovascular Surgery, Temple University School of Medicine, Philadelphia, $\mathrm{Pa}$.

Disclosures: Author has nothing to disclose with regard to commercial support.

Received for publication Nov 4, 2014; accepted for publication Nov 4, 2014; available ahead of print Dec 4, 2014.

Address for reprints: Grayson H. Wheatley III, MD, Temple University School of Medicine, 3401 N. Broad St, 3rd Floor, Zone C, Suite 301, Philadelphia, PA 19147 (E-mail: grayson.wheatley@tuhs.temple.edu).

J Thorac Cardiovasc Surg 2015;149:829-30

$0022-5223 / \$ 36.00$

Copyright (c) 2015 by The American Association for Thoracic Surgery

http://dx.doi.org/10.1016/j.jtcvs.2014.11.006
}

of 18 to 78 years) were included in the study. The mean age of the patients was $45.8 \pm 17$ years, and all patients were alive at the 10-year mark. More importantly, no incidences of graft migration, collapse, reintervention, or secondary endoleak had occurred. The proximal seal zone showed mild dilation, of $3.3 \pm 1.5 \mathrm{~mm}$, over the study period. Interestingly, a significantly greater increase in proximal neck diameter was found in those patients age $<30$ years, compared with the older patients: $P=.0034 ; 4 \pm 1.2 \mathrm{~mm}$ versus 1.5 $\pm 1.7 \mathrm{~mm}$. For the distal seal zone, a net $2.7 \pm 1 \mathrm{~mm}$ increase occurred during the study period. This increase did not reach statistical significance for the patients age $<30$ years versus older patients $(P=.065,3.3 \pm 1.6 \mathrm{~mm}$ vs $2.1 \pm 1.6 \mathrm{~mm})$.

This study helps answer several important questions surrounding the long-term management of TAT patients. Much of the long-term success in this study is predicated on excellent technical results at the time of the TEVAR procedure. As the authors appropriately note, minimal aortic stent oversizing (a maximum of $10 \%$ ) helps prevent graft infolding. Furthermore, appropriately dealing with the highly angulated aortic arch by deploying the aortic stentgraft at a suitable anatomic landing zone helps achieve seal and conformability of the aortic stent-graft. Lack of graft apposition on the underside of the aortic arch is an undesirable result that affects the durability of repair. To put the results of this study in perspective, the current generation of aortic stent-grafts has improved conformability and deployment mechanics compared with the original devices used at the time of treatment, which makes the technical results achieved even more impressive.

A gradual increase in aortic diameter at the proximal and distal landing zones of the aortic stent is to be expected, consistent with the natural history of the aorta. Before this study, the question of whether this diameter increase would have a clinical impact in TEVAR patients treated for TAT 